\title{
Old drug, new findings: colistin resistance and dependence of Acinetobacter baumannii
}

\author{
Kwan Soo Ko, Yujin Choi, Ji-Young Lee \\ Department of Molecular Cell Biology, Sungkyunkwan University School of Medicine, Suwon, Korea
}

Received: August 30, 2017

Revised: October 23, 2017

Accepted: October 24, 2017

Corresponding author:

Kwan Soo Ko

Department of Molecular

Cell Biology, Sungkyunkwan

University School of Medicine,

2066 Seobu-ro, Jangan-gu,

Suwon 16419, Korea

Tel: +82-31-299-6223

E-mail: ksko@skku.edu

\begin{abstract}
Colistin is an old drug, and its use has recently resurged because of increasing antibiotic resistance in gram-negative bacteria such as Acinetobacter baumannii. Although the colistin resistance rates in gram-negative bacteria are currently not high, many colistin-resistant isolates are being identified and the possibility of horizontal transmission of colistin resistance has increased because of the plasmid-borne colistin resistance gene mcr-1 (mobilized colistin resistance). In this review, we have discussed colistin resistance in $A$. baumannii. In addition, we have reviewed an abnormal phenomenon called colistin dependence in $A$. baumannii.
\end{abstract}

Keywords: Gram-negative bacteria; PmrAB; Polymyxins
This is an Open Access article distributed under the terms of the Creative Commons Attribution Non-Commercial License (http:// creativecommons.org/licenses/ by-nc/4.0/).

\section{INTRODUCTION}

Acinetobacter was most probably first described as Diplococcus mucosus in 1908. In 1954, Brisou and Prévot proposed the genus Acinetobacter to indicate that the bacteria were non-motile because they lacked flagella: the Greek "akineto" means "immobile" [1]. Acinetobacter spp. are glucose-non-fermentative, non-motile, non-fastidious, catalase-positive, oxidative-negative, aerobic, and gram-negative coccobacilli [2]. The genus Acinetobacter includes 55 species (as of July 18, 2017; http://www.bacterio.net/acinetobacter.html), and the number of species is increasing [3]. Acinetobacter baumannii is the most common species to cause infections, followed by Acinetobacter nosocomialis and Acinetobacter pittii [4,5]. Acinetobacter Iwoffii, Acinetobacter haemolyticus, Acinetobacter johnsonii, Acinetobacter junii, Acinetobacter ursingii, Acinetobacter schindleri, Acinetobacter calcoaceticus, and Acinetobacter seifertii have occasionally been reported in humans [5,6]. A. baumannii, A. calcoaceticus, A. nosocomialis, and $A$. pittii have very similar biochemical traits and could be separated well; they were grouped into the so-called "A. calcoaceticus-A. baumannii (Acb) complex" [7]. A. seifertii is also closely related to the species of the Acb complex [8,9].

Acinetobacter spp., including A. baumannii, have long been known as colonizers in humans, but they do not cause severe infections [10]. However, A. baumannii causes infections in immunosuppressed patients, patients with serious underlying diseases, and those subjected to invasive procedures and treated with broad-spectrum antibiotics; it may be a pathogen that 
has adapted the most to the hospital environment in the 21st century [11]. A. baumannii has become a representative pathogen that threatens human health. It is a member of the ESKAPE group, which is the main bacterial group that causes infections in humans [12], and has been recently ranked as a bacterium that poses the greatest health threat by World Health Organization [13]. In the United States, A. baumannii has been estimated to cause more than $2 \%$ of the healthcare-associated infections [14]. Acinetobacter spp. are the seventh most-isolated bacteria in Korean hospitals [15], and they are the most frequent isolates from adults with hospital-acquired pneumonia or ventilator-associated pneumonia in Asian countries, including South Korea [16].

Although $\beta$-lactam antibiotics are the preferred choice for susceptible $A$. baumannii infections, carbapenems have become the main therapeutic option because of an increase in resistance $[2,6]$. However, imipenem-resistant isolates were found in the early 1990s [17], and the rate of carbapenem resistance in A. baumannii, mainly due to OXA-type (oxacillin-hydrolysing) carbapenemases, has increased rapidly $[7,11]$. In South Korea, more than two-thirds of the A. baumannii isolates were resistant to imipenem on the basis of several surveillance studies $[4,15,18]$. Most of the carbapenem-resistant $A$. baumannii isolates showed multidrug resistance (MDR) or extreme drug resistance (XDR), which is defined as resistance to all available antibiotics, except for one or two agents [19]. Current treatment options for XDRA. baumannii infections remain quite limited. In addition to tigecycline, a recently developed antibiotic-an old drug, colistinis often the last resort for treating XDRA. baumannii $[6,20]$.

\section{COLISTIN}

Polymyxin antibiotics include colistin (also known as polymyxin $E$ ), and polymyxin $B$ is an antimicrobial polypeptide that was originally isolated in 1947 from the soil bacterium Paenibacillus polymyxa [21]. Colistin differs from polymyxin $B$ by only one amino acid at position 6 in the peptide ring: $a$ leucine in colistin and a phenylalanine in polymyxin $B$ [22]. Although they have similar antimicrobial spectra and resistance mechanisms, the method for administration is different: while polymyxin $B$ is administered directly in the active form, colistin is administered in the form of an inactive prodrug, colistin methanesulphonate (also known as colistimethate [CMS]). CMS itself lacks antibacterial activity, but it is converted into colistin after administration [23,24]. Although colistin has shown significant activity against a wide variety of gram-negative pathogens, its use was stopped in the 1970s because of its nephrotoxicity and neurotoxicity [25]. However, the emergence of MDR or carbapenem-resistant gram-negative bacterial pathogens and the lack of new antibiotics to treat them have led to the resurgence of colistin $[26,27]$.

Colistin mediates bactericidal activity by interacting with the lipid A component of the lipopolysaccharide (LPS) present on gram-negative pathogens, including $A$. baumannii [28]. Because of an electrostatic interaction between the positively charged colistin on one side and phosphate groups of the negatively charged lipid A membrane on the other side, divalent cations such as $\mathrm{Ca}^{2+}$ and $\mathrm{Mg}^{2+}$ are displaced from the membrane lipids. This destabilizes LPS and, consequently, increases the permeability of the membrane, leading to outer membrane disruption and cell death [26]. Other action mechanisms of colistin have been proposed: endotoxin effect, inhibition of vital respiratory enzymes, and hydroxyl radical production [28,29].

Colistin exhibits bactericidal activity in a concentration-dependent manner against gram-negative bacteria, including $A$. baumannii, with a minimal post-antibiotic effect [30]. However, re-growth with time has frequently observed [31], and the inoculum effect, a phenomenon of decreasing efficacy of an antibiotic with increasing bacterial density, has been reported [30]. Colistin has a relatively narrow in vitro bacteria-killing spectrum. It is active against gram-negative bacilli, such as Acinetobacter spp., Pseudomonas aeruginosa, Escherichia coli, Klebsiella spp., and Enterobacter spp. However, it has shown inactivity against some gram-negative bacilli, such as Burkholderia cepacia, Proteus spp., Providencia spp., and Serratia spp., as well as against gram-negative and gram-positive cocci, gram-positive bacilli, anaerobes, fungi, and parasites [32]. In addition, some Acinetobacter species, such as A. seifertii and Acinetobacter colistiniresistens, have exhibited very high colistin resistance rates or seem to be intrinsically resistant to it $[3,4]$.

\section{COLISTIN RESISTANCE}

Colistin resistance in gram-negative bacteria is known to occur via several mechanisms. The main mechanism is the addition of a cationic group, such as 4-amino-4-deoxy-L-arabinose (L-Ara4N) or phosphoethanolamine ( $\mathrm{pEtN}$ ) to the lipid A moiety of LPS, which results in a decrease in the net negative charge of the bacterial outer membrane [33-37]. In most gram-negative bacteria, the addition of cationic groups is 


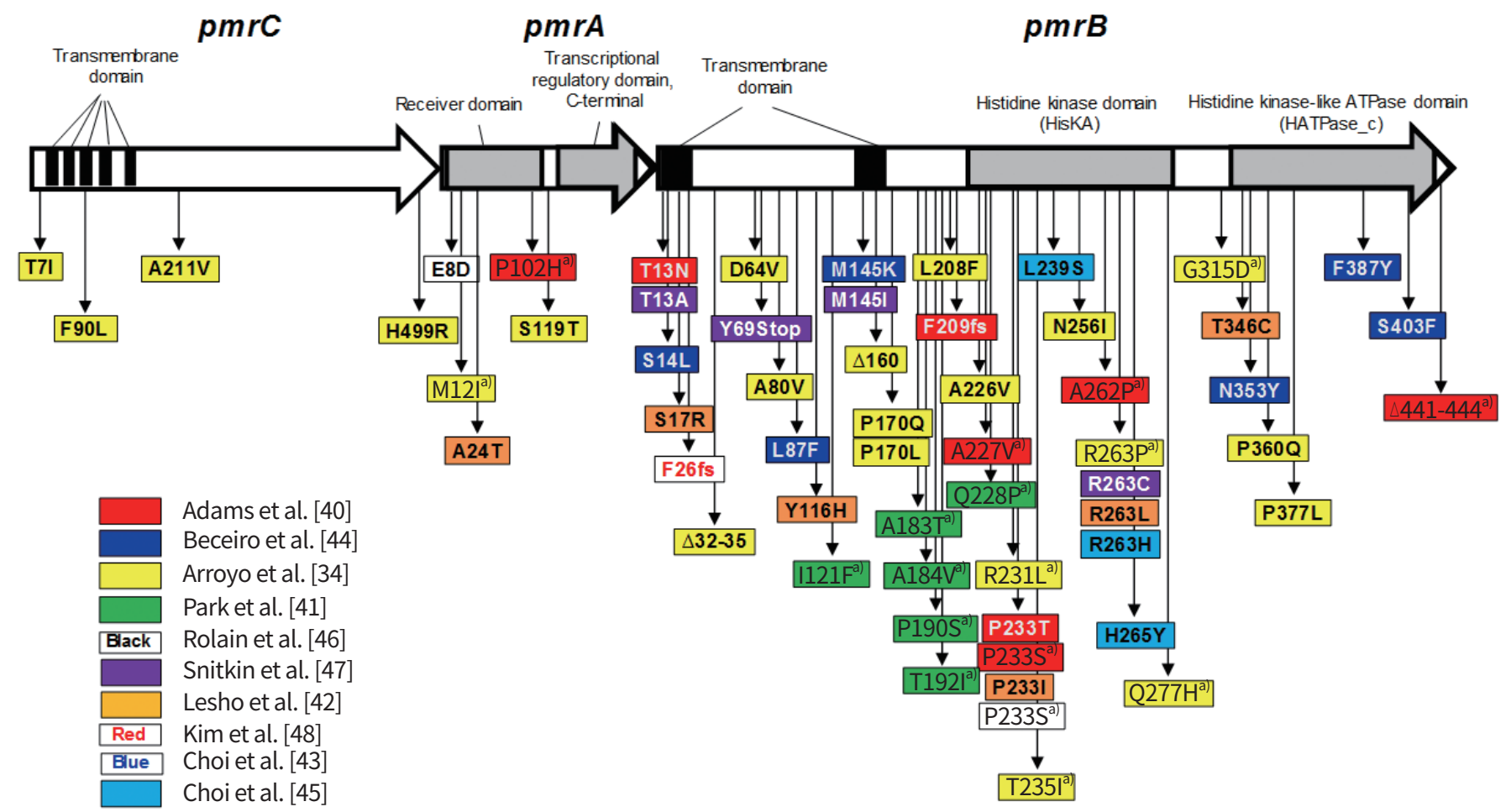

Fig. 1. Overview of amino acid substitutions associated with colistin resistance in the polymyxin resistance (pmr) operon in Acinetobacter baumannii. Amino acid alterations are indicated with different colors, according to the references. Location of domains within the pmr operon was predicted using the SMART (simple, modular, architecture, research, tool) program (http://smart.embl-heidelberg.de/). fs, frameshift; $\Delta$, deletion. ${ }^{a}$ On the top right of the letter indicates that an amino acid change found in vitro induced a colistin-resistant mutant.

regulated mainly by both $\mathrm{PhoPQ}$ and polymyxin resistance (pmr) PmrAB, which are two-component regulatory systems $[28,38]$. However, the phoPQ genes have not been found in the genome of Acinetobacter spp. [39]; thus, lipid A modification in $A$. baumannii is mediated by mutations in PmrAB $[33,34,40-43]$. Mutations in the $p m r A$ or $p m r B$ genes cause upregulation of the pmrCAB operon, leading to the synthesis and addition of $\mathrm{pEtN}$, which is responsible for colistin resistance in A. baumannii. Amino acid alternations in PmrCAB of A. baumannii reported to date are presented in Fig. 1 [34,4048]. As shown in Fig. 1, most amino acid substitutions associated with colistin resistance have been found in PmrB, a membrane-bound histidine kinase. However, it has not been verified experimentally if most variations are really responsible for colistin resistance in $A$. baumannii. Colistin-resistant mutants with no mutations in the pmrA and pmrB genes have also been identified, implying that the amino acid changes in the PmrAB two-component system are not essential for A. baumannii colistin resistance [41].

In addition to lipid A modification of LPS, loss of LPS has been reported to be associated with colistin resistance in $A$. baumannii [49]. Alterations in the lipid $A$ biosynthesis genes (IpxA, IpxC, and $I p \times D)$ by amino acid substitutions, deletions, or insertion of ISAba1 are responsible for the loss of LPS $[49,50]$. A recent metabolomic study revealed that an LPS-deficient, colistin-resistant $A$. baumannii strain showed perturbation in specific amino acid and carbohydrate metabolites, particularly pentose phosphate pathway and TCA (tricarboxylic acid) cycle intermediates [37]. In addition, depletion of peptidoglycan metabolites was observed in LPS-deficient strains. Several studies have reported increased susceptibility to some antibiotics rather than polymyxins in LPS-deficient, colistin-resistant $A$. baumannii strains [51,52], which has been postulated to be due to an increase in the passive diffusion of antibiotics. Decreased virulence in LPS-deficient strains has also been observed, which is compared with no change in the virulence of colistin-resistant strains due to pmrAB mutations [44,53]. To date, colistin resistance through the loss of LPS has not been detected in bacteria other than Acinetobacter spp.

Other colistin resistance mechanisms have been suggested in other gram-negative bacteria: overproduction of the cap- 
sule polysaccharide (CPS) and efflux pumps [54,55]. For CPS production, reduced production of CPS in colistin-resistant mutants was observed in Klebsiella pneumoniae, which is a contradictory finding [56]. While an efflux pump inhibitor, carbonyl cyanide 3-chlorophenylhydrazone (CCCP) increased colistin susceptibility in A. baumannii, other efflux pump inhibitors, including phenylalanine-arginine $\beta$-naphthylamide (PABN) did not show such an effect [31]. Thus, the roles of CPS overproduction and efflux pumps in colistin resistance should be further investigated.

Unlike the chromosome-related colistin resistance mechanisms described above, the plasmid-borne resistance gene mobilized colistin resistance ( $m c r-1$ ) has been recently reported from E. coli isolates in China [57]. Since the first report, $m c r-1$, which encodes $\mathrm{pEtN}$ transferase, has been detected in dozens of countries worldwide, including South Korea $[58,59]$. Although it has been reported in diverse bacterial species, such as E. coli, K. pneumoniae, Enterobacter cloacae, Enterobacter aerogenes, Salmonella spp., and Shigella sonnei, it has not been found in Acinetobacter spp. isolates [60]. However, a mcr-1-carrying plasmid could be introduced into $A$. baumannii, and reduced susceptibility to colistin was observed, highlighting the risk of horizontal transfer of colistin resistance in $A$. baumannii [61].

It is estimated that colistin preserves its activity against many gram-negative pathogens, including Acinetobacter spp. An antimicrobial susceptibility study based on worldwide collection during 2006 and 2009 exhibited that colistin showed potent in vitro activities against Acinetobacter spp. (MIC $\mathrm{M}_{90}, 1 \mathrm{mg} / \mathrm{L} ;$ 98.6\% susceptibility) [62]. Recent SENTRY Antimicrobial Surveillance Program data also show that more than $95 \%$ of Acinetobacter spp. isolates from Europe, China, and the United States are susceptible to colistin $[63,64]$. In South Korea, colistin resistance rates among $A$. baumannii isolates have been estimated to be $7.0 \%$ and $2.4 \%[4,8]$. A recent study has also shown a colistin resistance rate of $8.6 \%$ among Acinetobacter spp. clinical isolates [65]. However, it did not delineate Acinetobacter spp. and may have overestimated the colistin resistance rate in $A$. baumannii because of high colistin resistance rates in other species of the Acb complex, A. seifertii and $A$. pittii $[4,8]$. While colistin resistance in $A$. baumannii seems to occur readily by simple mutation in both laboratories and patients $[45,66]$, a genotyping study revealed that colistin-resistant $A$. baumannii isolates did not disseminate clonally [67].

\section{COLISTIN DEPENDENCE}

Several investigators have reported heteroresistance to colistin in A. baumannii, which has been supposed to cause the emergence of colistin resistance by exposure to colistin [6870]. Heteroresistance is generally defined as a case in which subpopulations of antibiotic-susceptible bacteria show resistance to certain antibiotics [71]. The heteroresistant subpopulations survive at high antibiotic concentrations in a ratio of $\sim 10^{-6}$ in a population analysis profiling (PAP) or appear as dis-

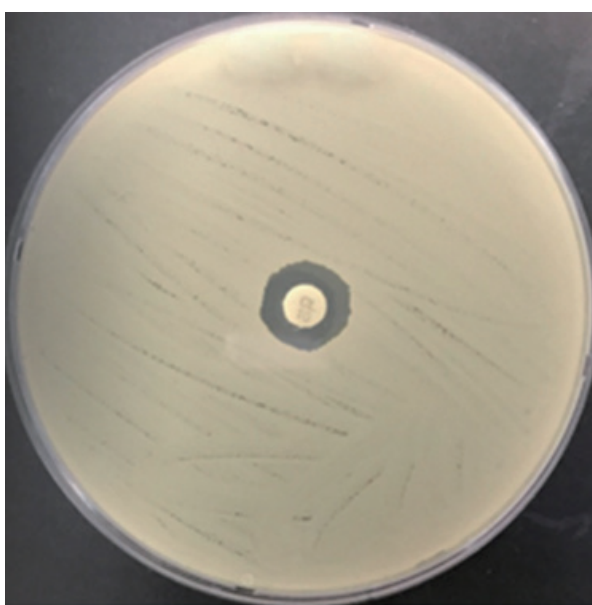

H06-855

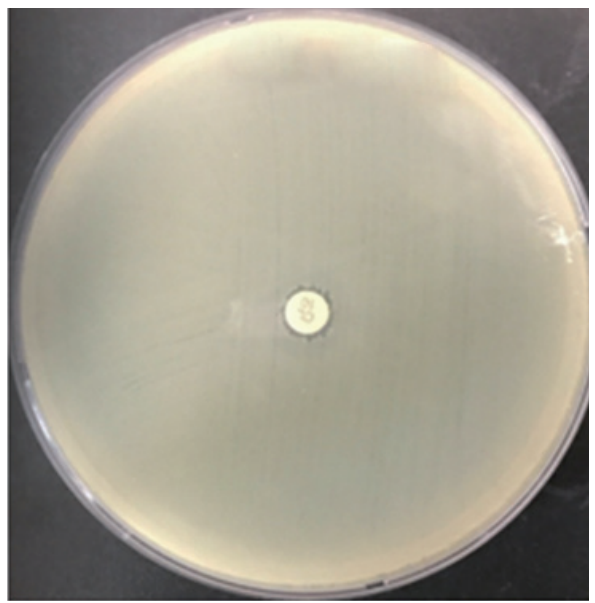

H06-855R

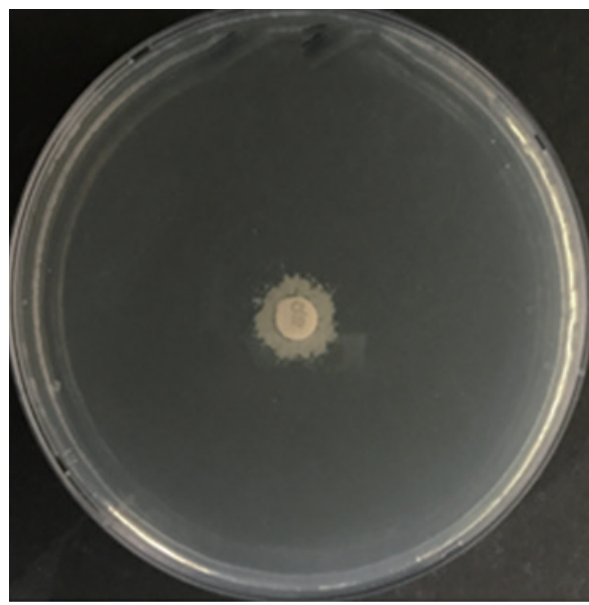

H06-855D

Fig. 2. Results of the disc diffusion assay for the colistin-susceptible, colistin-resistant, and colistin-dependent phenotypes. H06-855R and H06-855D are the colistin-resistant and colistin-dependent mutants, respectively, that originated from the colistin-susceptible Acinetobacter baumannii strain H06-855. The colistin-dependent mutant was obtained from colonies that survived $10 \mathrm{mg} / \mathrm{L}$ of colistin during the population analysis. While the colistin-resistant mutant grew throughout the plate, irrespective of the colistin disc, the colistin-dependent mutant grew only around the colistin disc. 
tinct colonies growing within the clear zone of inhibition in the disc or E-test assay [71].

Unusually, some surviving A. baumannii subpopulations at high colistin concentrations in PAP exhibit the colistin dependence phenomenon. That is, when surviving colonies at $\geq 8$ $\mathrm{mg} / \mathrm{L}$ colistin during PAP were plated on solid agar with discs of $10 \mathrm{mg}$ colistin, the bacteria grew only near the disc (Fig. 2). Such colistin dependence was first identified in an Acinetobacter $\mathrm{sp}$. isolate from a calcaneus bone specimen of a patient with calcaneal osteomyelitis and bacteremia by Hawley et al. [72] during a population analysis. It was the first report on colistin dependence, and they identified the isolate as $A$. baumannii-A. calcoaceticus because they did not identify it to the species level.

The antibiotic dependence phenomenon was first reported in vancomycin-dependent Enterococcus faecalis in 1994 [73]. Vancomycin dependence in enterococci has subsequently been identified [74-76]; it may not be rare and may occur regardless of the use of vancomycin [77]. Because vancomycin-dependent isolates lack ligase activity because of mutations in the D-alanine D-alanine ligase $(d d l)$ gene encoding the D-Ala-D-Ala ligase protein, they require glycopeptide antibiotics for cell-wall synthesis [78]. Although linezolid-dependent Staphylococcus epidermidis and $\beta$-lactam-dependent Staphylococcus saprophyticus have been reported $[79,80]$, antibiotic dependence has been rarely identified in gram-negative bacteria.

After Hawley et al. [72], Garcia-Quintanilla et al. [81] identified partial colistin dependence by using the E-test assay. They found that some LPS-deficient, colistin-resistant $A$. baumannii strains with mutations in IpxA, IpxC, and I $x$ D showed partial colistin dependence. However, colistin resistance through LPS modification due to mutations in the Pm$\mathrm{rAB}$ did not convert into colistin dependence. Although they proposed the loss of LPS as a colistin dependence mechanism, they did not address why a colistin-resistant isolate with the loss of LPS converted into colistin dependence. Thus, the mechanism underlying colistin dependence in $A$. baumannii is unclear.

Recently, we reported the development of colistin dependence in clinical colistin-susceptible $A$. baumannii isolates after exposure to colistin [82]. In that study, development of colistin dependence was not rare; $32.9 \%$ of 149 colistin-susceptible isolates developed colistin dependence. Genotypic analyses revealed that colistin dependence originated from the corresponding susceptible parental isolates, and no evidence of clonal dissemination of the isolates that developed colistin dependence was found. Colistin-dependent mutants have shown increased susceptibility to several antibiotics, such as carbapenems $[72,81,82]$, which is the feature of LPS-deficient, colistin-resistant isolates [51,52]. Of note, patients with colistin-dependent strains have shown higher 3and 7-day treatment failure than the patients without colistin-dependent strains [82]. Thus, the development of colistin-dependent mutants may have clinical significance, and it should be investigated. A recent study showed that the colistin-dependent phenotype may arise from the loss of LPS or defects in its structure, resulting from the disruption of LpxC [83]. In that study, transition of colistin dependence into colistin resistance was also demonstrated in the absence of antibiotic selection pressure [83].

\section{CONCLUSION}

The need for new antibiotics is growing in this era of antibiotic resistance; however, the development of new antibiotics, particularly for MDR gram-negative bacteria, has slowed down. Thus, the importance of older drugs, such as colistin, is increasing. However, the colistin resistance rate seems to be increasing, and information on the colistin resistance mechanism is limited. In addition to colistin resistance, abnormal phenomena such as colistin dependence have been found; however, there are few studies on colistin dependence. To cope with the antibiotic resistance era and use colistin effectively, a wide range of studies on colistin resistance and dependence should be performed.

\section{CONFLICTS OF INTEREST}

No potential conflict of interest relevant to this article was reported.

\section{REFERENCES}

1. Henry R. Etymologia: acinetobacter. Emerg Infect Dis 2013;19:841.

2. Bergogne-Berezin E, Towner KJ. Acinetobacter spp. as nosocomial pathogens: microbiological, clinical, and epidemiological features. Clin Microbiol Rev 1996;9:148-65.

3. Nemec A, Radolfova-Krizova L, Maixnerova M, Sedo O. Acinetobacter colistiniresistens sp. nov. (formerly genomic species 13 sensu Bouvet and Jeanjean and genomic species 14 sensu Tjernberg and Ursing), isolated from human infections and characterized by intrinsic re- 
PRECISION AND FUTURE MIEDICINE

Colistin resistance and dependence in $A$. baumannii

sistance to polymyxins. Int J Syst Evol Microbiol 2017;67: 2134-41.

4. Park YK, Jung SI, Park KH, Kim DH, Choi JY, Kim SH, et al. Changes in antimicrobial susceptibility and major clones of Acinetobacter calcoaceticus-baumannii complex isolates from a single hospital in Korea over 7 years. J Med Microbiol 2012;61(Pt 1):71-9.

5. Park KH, Shin JH, Lee SY, Kim SH, Jang MO, Kang SJ, et al. The clinical characteristics, carbapenem resistance, and outcome of Acinetobacter bacteremia according to genospecies. PLoS One 2013;8:e65026.

6. Wong D, Nielsen TB, Bonomo RA, Pantapalangkoor P, Luna B, Spellberg B. Clinical and pathophysiological overview of Acinetobacter infections: a century of challenges. Clin Microbiol Rev 2017;30:409-47.

7. Dijkshoorn L, Nemec A, Seifert H. An increasing threat in hospitals: multidrug-resistant Acinetobacter baumannii. Nat Rev Microbiol 2007;5:939-51.

8. Ko KS, Suh JY, Kwon KT, Jung SI, Park KH, Kang Cl, et al. High rates of resistance to colistin and polymyxin $B$ in subgroups of Acinetobacter baumannii isolates from Korea. J Antimicrob Chemother 2007;60:1163-7.

9. Kim DH, Park YK, Choi JY, Ko KS. Identification of genetic recombination between Acinetobacter species based on multilocus sequence analysis. Diagn Microbiol Infect Dis 2012;73:284-6.

10. Peleg AY, Seifert H, Paterson DL. Acinetobacter baumannii: emergence of a successful pathogen. Clin Microbiol Rev 2008;21:538-82.

11. Perez F, Hujer AM, Hujer KM, Decker BK, Rather PN, Bonomo RA. Global challenge of multidrug-resistant Acinetobacter baumannii. Antimicrob Agents Chemother 2007; 51:3471-84.

12. Boucher HW, Talbot GH, Bradley JS, Edwards JE, Gilbert D, Rice LB, et al. Bad bugs, no drugs: no ESKAPE! An update from the Infectious Diseases Society of America. Clin Infect Dis 2009;48:1-12.

13. Willyard C. The drug-resistant bacteria that pose the greatest health threats. Nature 2017;543:15.

14. Centres for Disease Control and Prevention (U.S.) Antibiotic resistance threats in the United States, 2013. Atlanta (GA): Centres for Disease Control and Prevention, U.S. Department of Health and Human Services; 2013.

15. Yong D, Shin HB, Kim YK, Cho J, Lee WG, Ha GY, et al. Increase in the prevalence of carbapenem-resistant Acinetobacter isolates and ampicillin-resistant non-typhoidal salmonella species in Korea: a KONSAR Study conducted in 2011. Infect Chemother 2014;46:84-93.

16. Chung DR, Song JH, Kim SH, Thamlikitkul V, Huang SG, Wang $\mathrm{H}$, et al. High prevalence of multidrug-resistant nonfermenters in hospital-acquired pneumonia in Asia. Am J Respir Crit Care Med 2011;184:1409-17.

17. Tankovic J, Legrand P, De Gatines G, Chemineau V, Brun-Buisson C, Duval J. Characterization of a hospital outbreak of imipenem-resistant Acinetobacter baumannii by phenotypic and genotypic typing methods. J Clin Microbiol 1994;32:2677-81.

18. Korea Centers for Disease Control and Prevention (KCDC). KARMS (Korean Antimicrobial Resistance Monitoring System) 2015 Annual Report. Cheongju (KR): KCDC, Korea National Institute of Health; 2016.

19. Magiorakos AP, Srinivasan A, Carey RB, Carmeli Y, Falagas ME, Giske CG, et al. Multidrug-resistant, extensively drug -resistant and pandrug-resistant bacteria: an international expert proposal for interim standard definitions for acquired resistance. Clin Microbiol Infect 2012;18:268-81.

20. Nation RL, Li J. Colistin in the 21st century. Curr Opin Infect Dis 2009;22:535-43.

21. Benedict RG, Langlykke AF. Antibiotic activity of Bacillus polymyxa. J Bacteriol 1947;54:24.

22. Velkov T, Thompson PE, Nation RL, Li J. Structure: activity relationships of polymyxin antibiotics. J Med Chem 2010; 53:1898-916.

23. Bergen PJ, Li J, Rayner CR, Nation RL. Colistin methanesulfonate is an inactive prodrug of colistin against Pseudomonas aeruginosa. Antimicrob Agents Chemother 2006; 50:1953-8.

24. Nation RL, Velkov T, Li J. Colistin and polymyxin B: peas in a pod, or chalk and cheese? Clin Infect Dis 2014;59:88-94.

25. Kelesidis T, Falagas ME. The safety of polymyxin antibiotics. Expert Opin Drug Saf 2015;14:1687-701.

26. Li J, Nation RL, Turnidge JD, Milne RW, Coulthard K, Rayner CR, et al. Colistin: the re-emerging antibiotic for multidrug-resistant Gram-negative bacterial infections. Lancet Infect Dis 2006;6:589-601.

27. Landman D, Georgescu C, Martin DA, Quale J. Polymyxins revisited. Clin Microbiol Rev 2008;21:449-65.

28. Poirel L, Jayol A, Nordmann P. Polymyxins: antibacterial activity, susceptibility testing, and resistance mechanisms encoded by plasmids or chromosomes. Clin Microbiol Rev 2017;30:557-96.

29. Sampson TR, Liu X, Schroeder MR, Kraft CS, Burd EM, Weiss DS. Rapid killing of Acinetobacter baumannii by polymyxins is mediated by a hydroxyl radical death path- 
way. Antimicrob Agents Chemother 2012;56:5642-9.

30. Tran TB, Velkov T, Nation RL, Forrest A, Tsuji BT, Bergen PJ, et al. Pharmacokinetics/pharmacodynamics of colistin and polymyxin B: are we there yet? Int J Antimicrob Agents 2016;48:592-7.

31. Park YK, Ko KS. Effect of carbonyl cyanide 3-chlorophenylhydrazone (CCCP) on killing Acinetobacter baumannii by colistin. J Microbiol 2015;53:53-9.

32. Falagas ME, Kasiakou SK. Colistin: the revival of polymyxins for the management of multidrug-resistant gram-negative bacterial infections. Clin Infect Dis 2005; 40:1333-41.

33. Beceiro A, Llobet E, Aranda J, Bengoechea JA, Doumith M, Hornsey M, et al. Phosphoethanolamine modification of lipid A in colistin-resistant variants of Acinetobacter baumannii mediated by the pmrAB two-component regulatory system. Antimicrob Agents Chemother 2011;55:33709.

34. Arroyo LA, Herrera CM, Fernandez L, Hankins JV, Trent MS, Hancock RE. The pmrCAB operon mediates polymyxin resistance in Acinetobacter baumannii ATCC 17978 and clinical isolates through phosphoethanolamine modification of lipid A. Antimicrob Agents Chemother 2011;55:3743-51.

35. Park YK, Lee JY, Ko KS. Transcriptomic analysis of colistin-susceptible and colistin-resistant isolates identifies genes associated with colistin resistance in Acinetobacter baumannii. Clin Microbiol Infect 2015;21:765.

36. Qureshi ZA, Hittle LE, O'Hara JA, Rivera JI, Syed A, Shields RK, et al. Colistin-resistant Acinetobacter baumannii: beyond carbapenem resistance. Clin Infect Dis 2015;60: 1295-303.

37. Maifiah MH, Cheah SE, Johnson MD, Han ML, Boyce JD, Thamlikitkul V, et al. Global metabolic analyses identify key differences in metabolite levels between polymyxin-susceptible and polymyxin-resistant Acinetobacter baumannii. Sci Rep 2016;6:22287.

38. Olaitan AO, Morand S, Rolain JM. Mechanisms of polymyxin resistance: acquired and intrinsic resistance in bacteria. Front Microbiol 2014;5:643.

39. Adams MD, Goglin K, Molyneaux N, Hujer KM, Lavender H, Jamison JJ, et al. Comparative genome sequence analysis of multidrug-resistant Acinetobacter baumannii. J Bacteriol 2008;190:8053-64.

40. Adams MD, Nickel GC, Bajaksouzian S, Lavender H, Murthy AR, Jacobs MR, et al. Resistance to colistin in Acinetobacter baumannii associated with mutations in the PmrAB two-component system. Antimicrob Agents Chemother 2009;53:3628-34.
41. Park YK, Choi JY, Shin D, Ko KS. Correlation between overexpression and amino acid substitution of the Pmr$A B$ locus and colistin resistance in Acinetobacter baumannii. Int J Antimicrob Agents 2011;37:525-30.

42. Lesho E, Yoon EJ, McGann P, Snesrud E, Kwak Y, Milillo M, et al. Emergence of colistin-resistance in extremely drug-resistant Acinetobacter baumannii containing a novel pmr$C A B$ operon during colistin therapy of wound infections. J Infect Dis 2013;208:1142-51.

43. Choi MJ, Ko KS. Mutant prevention concentrations of colistin for Acinetobacter baumannii, Pseudomonas aeruginosa and Klebsiella pneumoniae clinical isolates. J Antimicrob Chemother 2014;69:275-7.

44. Beceiro A, Moreno A, Fernandez N, Vallejo JA, Aranda J, Adler B, et al. Biological cost of different mechanisms of colistin resistance and their impact on virulence in Acinetobacter baumannii. Antimicrob Agents Chemother 2014; 58:518-26.

45. Choi HJ, Kil MC, Choi JY, Kim SJ, Park KS, Kim YJ, et al. Characterisation of successive Acinetobacter baumannii isolates from a deceased haemophagocytic lymphohistiocytosis patient. Int J Antimicrob Agents 2017;49:102-6.

46. Rolain JM, Diene SM, Kempf M, Gimenez G, Robert C, Raoult D. Real-time sequencing to decipher the molecular mechanism of resistance of a clinical pan-drug-resistant Acinetobacter baumannii isolate from Marseille, France. Antimicrob Agents Chemother 2013;57:592-6.

47. Snitkin ES, Zelazny AM, Gupta J; NISC Comparative Sequencing Program, Palmore TN, Murray PR, et al. Genomic insights into the fate of colistin resistance and Acinetobacter baumannii during patient treatment. Genome Res 2013;23:1155-62.

48. Kim Y, Bae IK, Lee H, Jeong SH, Yong D, Lee K. In vivo emergence of colistin resistance in Acinetobacter baumannii clinical isolates of sequence type 357 during colistin treatment. Diagn Microbiol Infect Dis 2014;79:362-6.

49. Moffatt JH, Harper M, Harrison P, Hale JD, Vinogradov E, Seemann T, et al. Colistin resistance in Acinetobacter baumannii is mediated by complete loss of lipopolysaccharide production. Antimicrob Agents Chemother 2010; 54:4971-7.

50. Moffatt JH, Harper M, Adler B, Nation RL, Li J, Boyce JD. Insertion sequence ISAba11 is involved in colistin resistance and loss of lipopolysaccharide in Acinetobacter baumannii. Antimicrob Agents Chemother 2011;55:30224.

51. Li J, Nation RL, Owen RJ, Wong S, Spelman D, Franklin C. 
PRECISION AND FUTURE MIEDICINE

Colistin resistance and dependence in $A$. baumannii

Antibiograms of multidrug-resistant clinical Acinetobacter baumannii: promising therapeutic options for treatment of infection with colistin-resistant strains. Clin Infect Dis 2007;45:594-8.

52. Mendes RE, Fritsche TR, Sader HS, Jones RN. Increased antimicrobial susceptibility profiles among polymyxin-resistant Acinetobacter baumannii clinical isolates. Clin Infect Dis 2008;46:1324-6.

53. Vila-Farres X, Ferrer-Navarro M, Callarisa AE, Marti S, Espinal P, Gupta S, et al. Loss of LPS is involved in the virulence and resistance to colistin of colistin-resistant Acinetobacter nosocomialis mutants selected in vitro. J Antimicrob Chemother 2015;70:2981-6.

54. Llobet E, Tomas JM, Bengoechea JA. Capsule polysaccharide is a bacterial decoy for antimicrobial peptides. Microbiology 2008;154(Pt 12):3877-86.

55. Srinivasan VB, Rajamohan G. KpnEF, a new member of the Klebsiella pneumoniae cell envelope stress response regulon, is an SMR-type efflux pump involved in broad -spectrum antimicrobial resistance. Antimicrob Agents Chemother 2013;57:4449-62.

56. Choi MJ, Ko KS. Loss of hypermucoviscosity and increased fitness cost in colistin-resistant Klebsiella pneumoniae sequence type 23 strains. Antimicrob Agents Chemother 2015;59:6763-73.

57. Liu YY, Wang Y, Walsh TR, Yi LX, Zhang R, Spencer J, et al. Emergence of plasmid-mediated colistin resistance mechanism MCR-1 in animals and human beings in China: a microbiological and molecular biological study. Lancet Infect Dis 2016;16:161-8.

58. Lim SK, Kang HY, Lee K, Moon DC, Lee HS, Jung SC. First detection of the mcr-1 gene in Escherichia coli isolated from livestock between 2013 and 2015 in South Korea. Antimicrob Agents Chemother 2016;60:6991-3.

59. Kim ES, Chong YP, Park SJ, Kim MN, Kim SH, Lee SO, et al. Detection and genetic features of MCR-1-producing plasmid in human Escherichia coli infection in South Korea. Diagn Microbiol Infect Dis 2017;89:158-60.

60. Jeannot K, Bolard A, Plesiat P. Resistance to polymyxins in Gram-negative organisms. Int J Antimicrob Agents 2017; 49:526-35.

61. Liu YY, Chandler CE, Leung LM, McElheny CL, Mettus RT, Shanks RMQ, et al. Structural modification of lipopolysaccharide conferred by mcr-1 in gram-negative ESKAPE pathogens. Antimicrob Agents Chemother 2017;61.

62. Gales AC, Jones RN, Sader HS. Contemporary activity of colistin and polymyxin B against a worldwide collection of Gram-negative pathogens: results from the SENTRY Antimicrobial Surveillance Program (2006-09). J Antimicrob Chemother 2011;66:2070-4.

63. Sader HS, Farrell DJ, Flamm RK, Jones RN. Antimicrobial susceptibility of Gram-negative organisms isolated from patients hospitalized in intensive care units in United States and European hospitals (2009-2011). Diagn Microbiol Infect Dis 2014;78:443-8.

64. Flamm RK, Nichols WW, Sader HS, Farrell DJ, Jones RN. In vitro activity of ceftazidime/avibactam against Gramnegative pathogens isolated from pneumonia in hospitalised patients, including ventilated patients. Int J Antimicrob Agents 2016;47:235-42.

65. Park GE, Kang Cl, Cha MK, Cho SY, Seok H, Lee JH, et al. Bloodstream infections caused by Acinetobacter species with reduced susceptibility to tigecycline: clinical features and risk factors. Int J Infect Dis 2017;62:26-31.

66. Lee JY, Choi MJ, Choi HJ, Ko KS. Preservation of acquired colistin resistance in gram-negative bacteria. Antimicrob Agents Chemother 2015;60:609-12.

67. Park YK, Jung SI, Park KH, Cheong HS, Peck KR, Song JH, et al. Independent emergence of colistin-resistant Acinetobacter spp. isolates from Korea. Diagn Microbiol Infect Dis 2009;64:43-51.

68. Li J, Rayner CR, Nation RL, Owen RJ, Spelman D, Tan KE, et al. Heteroresistance to colistin in multidrug-resistant Acinetobacter baumannii. Antimicrob Agents Chemother 2006;50:2946-50.

69. Hawley JS, Murray CK, Jorgensen JH. Colistin heteroresistance in acinetobacter and its association with previous colistin therapy. Antimicrob Agents Chemother 2008; 52: 351-2.

70. Yau W, Owen RJ, Poudyal A, Bell JM, Turnidge JD, Yu HH, et al. Colistin hetero-resistance in multidrug-resistant Acinetobacter baumannii clinical isolates from the Western Pacific region in the SENTRY antimicrobial surveillance programme. J Infect 2009;58:138-44.

71. El-Halfawy OM, Valvano MA. Antimicrobial heteroresistance: an emerging field in need of clarity. Clin Microbiol Rev 2015;28:191-207.

72. Hawley JS, Murray CK, Jorgensen JH. Development of colistin-dependent Acinetobacter baumannii-Acinetobacter calcoaceticus complex. Antimicrob Agents Chemother 2007;51:4529-30.

73. Fraimow HS, Jungkind DL, Lander DW, Delso DR, Dean JL. Urinary tract infection with an Enterococcus faecalis isolate that requires vancomycin for growth. Ann Intern 
Med 1994;121:22-6.

74. Van Bambeke F, Chauvel M, Reynolds PE, Fraimow HS, Courvalin P. Vancomycin-dependent Enterococcus faecalis clinical isolates and revertant mutants. Antimicrob Agents Chemother 1999;43:41-7.

75. Kirkpatrick BD, Harrington SM, Smith D, Marcellus D, Miller C, Dick J, et al. An outbreak of vancomycin-dependent Enterococcus faecium in a bone marrow transplant unit. Clin Infect Dis 1999;29:1268-73.

76. Tambyah PA, Marx JA, Maki DG. Nosocomial infection with vancomycin-dependent enterococci. Emerg Infect Dis 2004;10:1277-81.

77. Hwang K, Sung H, Namgoong S, Yoon NS, Kim MN. Microbiological and epidemiological characteristics of vancomycin-dependent enterococci. Korean J Lab Med 2009; 29:299-306.

78. Kuo SF, Huang SP, Lee CH. Vancomycin-dependent Enterococcus faecium can easily be obscured. J Microbiol Immunol Infect 2016.

79. Worthington T, White J, Lambert P, Adlakha S, Elliott T.
Beta-lactam-dependent coagulase-negative staphylococcus associated with urinary-tract infection. Lancet 1999;354:1097.

80. Pournaras S, Ntokou E, Zarkotou O, Ranellou K, Themeli-Digalaki K, Stathopoulos C, et al. Linezolid dependence in Staphylococcus epidermidis bloodstream isolates. Emerg Infect Dis 2013;19:129-32.

81. Garcia-Quintanilla M, Carretero-Ledesma M, Moreno-Martinez P, Martin-Pena R, Pachon J, McConnell MJ. Lipopolysaccharide loss produces partial colistin dependence and collateral sensitivity to azithromycin, rifampicin and vancomycin in Acinetobacter baumannii. Int J Antimicrob Agents 2015;46:696-702.

82. Hong YK, Lee JY, Wi YM, Ko KS. High rate of colistin dependence in Acinetobacter baumannii. J Antimicrob Chemother 2016;71:2346-8.

83. Lee JY, Chung ES, Ko KS. Transition of colistin dependence into colistin resistance in Acinetobacter baumannii. Sci Rep 2017;7:14216. 